\title{
Dynamic ray tracing and traveltime corrections for global seismic tomography
}

\author{
Yue Tian ${ }^{\mathrm{a}, *}$, S.-H. Hung ${ }^{\mathrm{b}}$, Guust Nolet ${ }^{\mathrm{a}}$, Raffaella Montelli ${ }^{\mathrm{c}}$, F.A. Dahlen ${ }^{\mathrm{a}}$ \\ a Department of Geosciences, Guyot Hall, Princeton University, NJ 08544, USA \\ ${ }^{\mathrm{b}}$ Department of Geosciences, National Taiwan University, Taipei 106, Taiwan \\ c ExxonMobil Upstream Research Company, P.O. Box 22189, GW03-940A, Houston, TX 77252-2189, USA
}

Received 9 February 2007; received in revised form 20 April 2007; accepted 24 April 2007

Available online 22 May 2007

\begin{abstract}
We present a dynamic ray tracing program for a spherically symmetric Earth that may be used to compute Fréchet kernels for traveltime and amplitude anomalies at finite frequency. The program works for arbitrarily defined phases and background models. The numerical precisions of kinematic and dynamic ray tracing are optimized to produce traveltime errors under $0.1 \mathrm{~s}$, which is well below the data uncertainty in global seismology. This tolerance level is obtained for an integration step size of about $20 \mathrm{~km}$ for the most common seismic phases. We also give software to compute ellipticity, crustal and topographic corrections and attenuation.
\end{abstract}

(C) 2007 Elsevier Inc. All rights reserved.

PACS: 02.60.-x; 91.30.-f; 91.30.Ab; 91.30.Cd; 91.35.Pn

Keywords: Finite-frequency tomography; Dynamic ray tracing; Traveltime correction; Computational seismology; Numerical precision

\section{Introduction}

Global tomography studies using seismic body waves such as $P$ and $S$ were so far mostly based on ray theory, a high-frequency approximation to the elastodynamic wave equation. Ray theory ignores wave scattering and wavefront healing effects, which render the traveltime anomalies dependent on the Earth structure in a 3D region around the geometrical ray, rather than limiting the sensitivity to an infinitesimally narrow ray path. Recently, Dahlen et al. [4] formulated an efficient theory for 3D traveltime sensitivity (or Fréchet) kernels, using a paraxial approximation and dynamic ray tracing [19] to reduce the computational effort. The ray tracing software that formed the starting point for the program described in this paper was written by Hung $[7,8]$ and applied by Montelli et al. $[13,14,12]$ in global inversions of delay times of compressional and shear waves.

\footnotetext{
* Corresponding author. Tel.: +1 609258 1504; fax: +1 6092581274.

E-mail address: ytian@princeton.edu (Y. Tian).
} 
The program described in this paper is a largely rewritten version of the earlier software. We have extended the versatility of the code by allowing seismic phases and the background model to be defined arbitrarily by the user. We have also added corrections for crustal structure, topography and ellipticity, and added additional output, such as $t^{*}$. The resulting program raydyntrace.f is available from http://geodynamics.org/cig/software/packages/.

Extensive comments and documentation have been added to make the code user-friendly. In this paper we describe the theory and present some results of the tests used to validate the code.

\section{Overview}

In finite-frequency tomography, the linearized relationship between the observed delay of compressional waves $\delta T$, and the relative deviations $\delta c_{P} / c_{P}$ in the compressional velocity is given by a volume integral [4]:

$$
\delta T=\int_{V} K_{P}\left(\boldsymbol{r}_{x}\right) \frac{\delta c_{P}}{c_{P}} \mathrm{~d}^{3} \boldsymbol{r}_{x}
$$

Theoretically, the integration volume $V$ is over the whole Earth. In practice, $V$ is the volume in which $\delta c_{P} / c_{P}$ has an observable contribution to $\delta T$, and is approximately the 3D Fresnel volume in the vicinity of the geometrical ray. $K_{P}\left(\boldsymbol{r}_{x}\right)$ is the Fréchet or 'banana-doughnut' kernel at a scatterer point $\boldsymbol{r}_{x}$ :

$$
K_{P}\left(\boldsymbol{r}_{x}\right)=-\frac{1}{2 \pi c_{P}\left(\boldsymbol{r}_{r}\right) c_{P}\left(\boldsymbol{r}_{x}\right)} \frac{\mathscr{R}_{r s}}{\mathscr{R}_{x r} \mathscr{R}_{x s}} \frac{\mathscr{P}_{s}^{P}}{\mathscr{P}^{P}} \times \frac{\int_{0}^{\infty} \omega^{3}|\dot{m}(\omega)|^{2} \sin \left[\omega \Delta T\left(\boldsymbol{r}_{x}\right)-\Delta \Phi\left(\boldsymbol{r}_{x}\right)\right] \mathrm{d} \omega}{\int_{0}^{\infty} \omega^{2}|\dot{m}(\omega)|^{2} \mathrm{~d} \omega},
$$

where $\dot{m}(\omega)$ is the spectrum of the observed compressional waveform, $\mathscr{P}^{P}$ and $\mathscr{P}_{s}^{P}$ are the source radiation patterns for the direct ray and the ray to scatterer, respectively, and $\Delta \Phi$ is the phase shift due to passage through caustics or supercritical reflection. The kernel requires computation of the traveltimes from source $s$ and receiver $r$ to the scatterer $x$ to obtain the 'detour traveltime' $\Delta T=T_{x s}+T_{x r}-T_{r s}$, and the geometrical spreading factors $\mathscr{R}_{r s}, \mathscr{R}_{x s}$ and $\mathscr{R}_{x r}$, where the subscripts $r s, x s$ and $x r$ represent the ray paths from source to receiver, source to scatterer, and receiver to scatterer, respectively. $\Delta T$ and $\mathscr{R}$ are needed for every possible ray path $r s, x s$ and $x r$. The 'background' model, for which these quantities are evaluated must be piecewise smooth, such that ray theory is valid. If not, (2) cannot be used, but kernels can still be computed using finite-difference or spectral element techniques [17]. Very similar expressions exist for amplitudes [3], and the kernels for shear waves are obtained by replacing $c_{P}, \mathscr{R}, \mathscr{P}$ and $\Delta T$ by the equivalent quantities for shear waves.

To compute $\mathscr{R}$ and $\Delta T$, we first determine the direction in which the ray departs from the source, which can in principle be done using kinematic ray tracing. For a three-dimensional background model it requires the determination of the two angles that determine the ray direction at the source. Doing this by trial-and-error ('shooting') is notoriously difficult. Nolet et al. [15] use a combination of graph-theoretical, ray-bending and dynamic ray tracing algorithms to compute the detour time and geometrical spreading in local models, but such techniques are too inefficient to be used on a global scale. We therefore restrict ourselves to a spherically symmetric background Earth model. This not only simplifies the equations, but also reduces the problem of finding the correct ray direction to the determination of just one angle or, equivalently, a ray parameter.

There are essentially two methods to compute the kernels in a spherically symmetric Earth. Calvet and Chevrot [1] set up a table of traveltimes and geometrical spreading for sources at every depth between the surface and the Earth's center, or deepest structure of interest, and epicentral distance traveled. Because of the amplitude reciprocity

$$
c_{P}\left(\boldsymbol{r}_{x}\right) \mathscr{R}_{r x}=c_{P}\left(\boldsymbol{r}_{r}\right) \mathscr{R}_{x r},
$$

this is sufficient to compute all necessary geometrical spreading factors by interpolation. An alternative, used in this paper, is to avoid interpolation and use a paraxial approach with dynamic ray tracing. It has the advantage that all quantities are quickly available once the dynamic ray tracing has been performed, and requires virtually no extra storage. The disadvantage is the introduction of the paraxial approximation, which assumes that the wavefront near the direct ray has a parabolic shape. We also assume that the geometrical spreading $\mathscr{R}$ 
computed for the geometrical ray is constant over the wavefront in the neighbourhood of the ray. More specifically, we approximate $\Delta T$ and $\mathscr{R}$ using only information computed for the geometrical ray [4]:

$$
\begin{aligned}
& \Delta T=\frac{1}{2} \boldsymbol{q}^{T}\left(\boldsymbol{H}_{x s}+\boldsymbol{H}_{x r}\right) \boldsymbol{q}, \\
& \ln \mathscr{R}_{x s}^{2}=\int_{0}^{l} c \operatorname{tr}\left(\boldsymbol{H}_{l^{\prime} s}\right) \mathrm{d} l^{\prime}, \quad \ln \mathscr{R}_{x r}^{2}=\int_{L}^{l} c \operatorname{tr}\left(\boldsymbol{H}_{l^{\prime} r}\right) \mathrm{d} l^{\prime},
\end{aligned}
$$

where $\boldsymbol{q}=\left(q_{1}, q_{2}\right)^{T}$ and $l$ are ray centered coordinates of scatterer $x$ (Fig. 1), and $c$ is the seismic velocity. $\boldsymbol{H}_{x s}$ and $\boldsymbol{H}_{x r}$ are $2 \times 2$ Hessian matrices from source and receiver, respectively, with

$$
H_{i j}=\left.\frac{\partial^{2} T}{\partial q_{i} \partial q_{j}}\right|_{\boldsymbol{q}=\mathbf{0}} .
$$

$\boldsymbol{H}_{l^{\prime} s}$ and $\boldsymbol{H}_{l^{\prime} r}$ are evaluated at $\left(l^{\prime}, 0,0\right)$ along the ray path $0 \leqslant l^{\prime} \leqslant L \cdot \operatorname{tr}(\cdot)$ denotes the trace of the matrix. The forward and backward Hessian matrices are obtained by solving the corresponding Riccati equations [19]:

$$
\frac{\mathrm{d} \boldsymbol{H}_{x s}}{\mathrm{~d} l}+c \boldsymbol{H}_{x s} \boldsymbol{H}_{x s}=-\frac{1}{c^{2}} \boldsymbol{V}_{x}, \quad-\frac{\mathrm{d} \boldsymbol{H}_{x r}}{\mathrm{~d} l}+c \boldsymbol{H}_{x r} \boldsymbol{H}_{x r}=-\frac{1}{c^{2}} \boldsymbol{V}_{x},
$$

where $\boldsymbol{V}_{x}$ is a $2 \times 2$ matrix with $V_{i j}=\partial^{2} c / \partial q_{i} \partial q_{j}$ evaluated at $(l, 0,0)$.

Thus, we only need to do the forward and backward ray tracing on the geometrical ray to obtain $\Delta T$ and $\mathscr{R}$.

\section{Numerical procedures}

\subsection{Hessian matrices}

In this section, we describe the numerical procedure to solve the Riccati equations (6) in a spherically symmetric Earth, taking the forward (first) Riccati equation in (6) as an example. In order to solve it, we need the initial condition

$$
\boldsymbol{H}_{x s} \rightarrow \frac{1}{c_{s} l} \boldsymbol{I} \quad \text { as } l \rightarrow 0,
$$

where $c_{s}$ is the velocity at source, $l$ is shown in Fig. 1, and $\boldsymbol{I}$ is the identity matrix. A convenient numerical scheme to deal with the non-linearity of (6), as well as the divergence at source (see (7)), is to decompose $\boldsymbol{H}_{x s}$ as [18]

$$
\boldsymbol{H}_{x s}=\boldsymbol{P} \boldsymbol{Q}^{-1} \text {. }
$$

Substituting (8) into the first equation in (6), we get a first-order system:

$$
\frac{\mathrm{d} \boldsymbol{P}}{\mathrm{d} l}=-\frac{1}{c^{2}} \boldsymbol{V}_{x} \boldsymbol{Q}, \quad \frac{\mathrm{d} \boldsymbol{Q}}{\mathrm{d} l}=c \boldsymbol{P} .
$$

Accordingly, the initial condition (7) becomes

$$
\boldsymbol{P}(l=0)=\boldsymbol{I}, \quad \boldsymbol{Q}(l=0)=\mathbf{0} .
$$

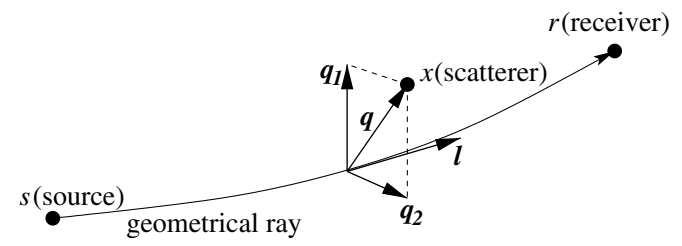

Fig. 1. Ray centered coordinates of scatterer $\boldsymbol{r}_{x}=\left(l, q_{1}, q_{2}\right)^{T}$, where $0 \leqslant l \leqslant L$ is the length of the geometrical ray measured from source. Vectors $\boldsymbol{l}, \boldsymbol{q}_{1}$ and $\boldsymbol{q}_{2}$ are mutually perpendicular, with $\boldsymbol{l}$ and $\boldsymbol{q}_{1}$ in the source-receiver plane. 
Because of the spherical symmetry, the differentiation with respect to $q_{2}$ always gives zero. Therefore $\boldsymbol{H}_{x s}$ is diagonal, and so are the $\boldsymbol{P}, \boldsymbol{Q}$ in (8):

$$
\boldsymbol{P}=\left(\begin{array}{cc}
P_{1} & 0 \\
0 & P_{2}
\end{array}\right), \quad \boldsymbol{Q}=\left(\begin{array}{cc}
Q_{1} & 0 \\
0 & Q_{2}
\end{array}\right) .
$$

From (8):

$$
\boldsymbol{H}_{x s}=\left(\begin{array}{cc}
P_{1} / Q_{1} & 0 \\
0 & P_{2} / Q_{2}
\end{array}\right) .
$$

To solve for $P_{1}, P_{2}, Q_{1}, Q_{2}$ from (9) and (10), we use a polar coordinate system in the source-receiver plane and change the independent variable from $l$ to $\phi$ (Fig. 2). Using the relations $\mathrm{d} \phi / \mathrm{d} l=\sin i / r$ and $p=r \sin i / c$, the first-order system (9) becomes

$$
\begin{aligned}
& \frac{\mathrm{d} P_{1}}{\mathrm{~d} \phi}=-\frac{p}{c}\left(\frac{\partial^{2} c}{\partial r^{2}}+\frac{1}{r} \frac{\partial c}{\partial r} \cot ^{2} i\right) Q_{1}, \quad \frac{\mathrm{d} P_{2}}{\mathrm{~d} \phi}=-\frac{r}{p c^{3}} \frac{\partial c}{\partial r} Q_{2} \\
& \frac{\mathrm{d} Q_{1}}{\mathrm{~d} \phi}=\frac{r^{2}}{p} P_{1}, \quad \frac{\mathrm{d} Q_{2}}{\mathrm{~d} \phi}=\frac{r^{2}}{p} P_{2}
\end{aligned}
$$

the initial conditions (10) become

$$
P_{1}(\phi=0)=P_{2}(\phi=0)=1, \quad Q_{1}(\phi=0)=Q_{2}(\phi=0)=0 ;
$$

and the continuity conditions are

$$
\begin{aligned}
& {\left[\cos i P_{1}+\left(\frac{p^{2}}{r^{2}} \frac{\partial c}{\partial r}-\frac{1}{r c}\right) Q_{1}\right]_{-}^{+}=0} \\
& {\left[P_{2}-\frac{\cos i}{r c} Q_{2}\right]_{-}^{+}=0, \quad\left[\frac{Q_{1}}{\cos i}\right]_{-}^{+}=\left[Q_{2}\right]_{-}^{+}=0,}
\end{aligned}
$$

where + and - represent the evaluation on the outgoing and incoming rays at the discontinuity depth, respectively. A fourth-order Runge-Kutta method is used to solve the first-order ODE system (12)-(14).

In a similar way, we can solve the backward (second) Riccati equation in (6) for $\boldsymbol{H}_{x r}$. Thus, we have reduced the kinematic two-point ray tracing to be only on the forward (from source to receiver) and backward (from receiver to source) geometrical rays.

\subsection{Avoiding backward two-point ray tracing}

Dahlen et al. [4] point out that some extra efficiency can be obtained by avoiding the backward two-point ray tracing and computing $\boldsymbol{H}_{x r}$ while doing the forward ray tracing. Here we give the results necessary for the numerical procedure.

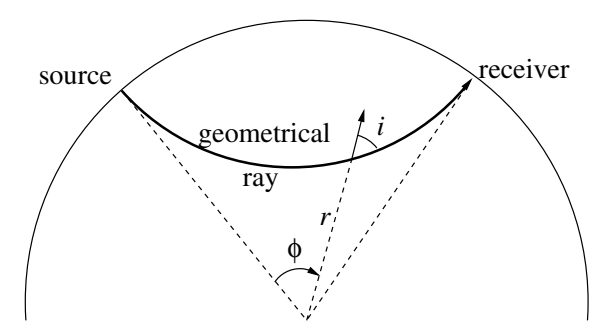

Fig. 2. Polar coordinates $\boldsymbol{r}=(r, \phi)$ in the source-receiver plane. $\phi=0$ at source and $\phi=\Delta$ at receiver. The incident angle $i$ is larger than $\pi / 2$ on the downgoing leg and smaller than $\pi / 2$ on the upgoing leg. 
Introduce a propagator matrix

$$
\mathscr{P}(\phi, 0)=\left(\begin{array}{cccc}
\widetilde{Q}_{1}(\phi) & 0 & Q_{1}(\phi) & 0 \\
0 & \widetilde{Q}_{2}(\phi) & 0 & Q_{2}(\phi) \\
\widetilde{P}_{1}(\phi) & 0 & P_{1}(\phi) & 0 \\
0 & \widetilde{P}_{2}(\phi) & 0 & P_{2}(\phi)
\end{array}\right),
$$

where $\widetilde{P}_{1}, \widetilde{P}_{2}, \widetilde{Q}_{1}, \widetilde{Q}_{2}$ satisfy (12) and (14) with different initial conditions

$$
\widetilde{P}_{1}(0)=\widetilde{P}_{2}(0)=0, \quad \widetilde{Q}_{1}(0)=\widetilde{Q}_{2}(0)=1 .
$$

Conditions (15) ensure that $\mathscr{P}$ is a propagator matrix because $\mathscr{P}(0,0)=\boldsymbol{I}$, and the columns of $\mathscr{P}$ are solutions to (12). We can thus express any solution as

$$
\boldsymbol{H}_{x s}(\phi)=\mathscr{P}(\phi, 0) \boldsymbol{H}_{x s}(0), \quad \boldsymbol{H}_{x r}(\phi)=\mathscr{P}(\phi, \Delta) \boldsymbol{H}_{x s}(\Delta),
$$

with $\phi=0$ at source and $\phi=\Delta$ at receiver. The backward propagator $\mathscr{P}(\phi, \Delta)$ can be obtained from the forward propagator $\mathscr{P}(\phi, 0)$ :

$$
\mathscr{P}(\phi, \Delta)=\mathscr{P}^{-1}(\Delta, \phi)=(\mathscr{P}(\Delta, 0) \mathscr{P}(0, \phi))^{-1}=\mathscr{P}(\phi, 0) \mathscr{P}^{-1}(\Delta, 0) .
$$

The final result can be written as

$$
\boldsymbol{H}_{x r}=\left(\begin{array}{cc}
P_{3} / Q_{3} & 0 \\
0 & P_{4} / Q_{4}
\end{array}\right),
$$

and $P_{3}, P_{4}, Q_{3}, Q_{4}$ are given by

$$
\begin{aligned}
P_{3}(\phi) & =P_{1}(\phi) \widetilde{Q}_{1}(\Delta)-\widetilde{P}_{1}(\phi) Q_{1}(\Delta), \\
P_{4}(\phi) & =P_{2}(\phi) \widetilde{Q}_{2}(\Delta)-\widetilde{P}_{2}(\phi) Q_{2}(\Delta), \\
Q_{3}(\phi) & =\widetilde{Q}_{1}(\phi) Q_{1}(\Delta)-Q_{1}(\phi) \widetilde{Q}_{1}(\Delta), \\
Q_{4}(\phi) & =\widetilde{Q}_{2}(\phi) Q_{2}(\Delta)-Q_{2}(\phi) \widetilde{Q}_{2}(\Delta) .
\end{aligned}
$$

To summarize, we have reduced the problem to one such that only one forward two-point ray tracing is needed for each source-receiver pair. To obtain the information near the geometrical ray, we solve Eqs. (12) and (14) with initial conditions (10) and (15) for $\left(P_{1}, Q_{1}\right),\left(P_{2}, Q_{2}\right),\left(\widetilde{P}_{1}, \widetilde{Q}_{1}\right),\left(\widetilde{P}_{2}, \widetilde{Q}_{2}\right)$, and obtain the Hessian matrices $\boldsymbol{H}_{x s}$ and $\boldsymbol{H}_{x r}$ by (11) and (16), respectively. Finally, one can get the detour time from (3).

One difficulty to be expected is the numerical instability caused by the divergence of $\boldsymbol{H}$ near source and receiver. As $Q_{1} \rightarrow 0$, small errors in $Q_{1}$ blow up to a large error in $\boldsymbol{H}_{x s}$. In fact, the initial conditions (13) make $\boldsymbol{H}_{x s}$ infinite at source; and (17) indicates that $\boldsymbol{H}_{x r}$ is infinite at receiver. To solve this problem, we assume a small homogeneous sphere around source or receiver and apply condition (7) in that sphere. A similar numerical fix could be used at other points where $Q_{1}$ or $Q_{3} \rightarrow 0$, such as near caustics for the PP wave, but we have not seen an urgent need for that in computations done so far.

Note that $\Delta T \rightarrow 0$ as one approaches source or receiver, so that the integrand in (2) remains finite (but multivalued in their singular points themselves).

\subsection{Geometrical spreading}

Further efficiency can be obtained by avoiding computing individual geometrical spreading factors from (4). In the traveltime and amplitude kernels, the geometrical spreading factors appear in the form of $\mathscr{R}_{r s} / \mathscr{R}_{x s} \mathscr{R}_{x r}$, and this term can be obtained from the determinant of the Hessian matrices $[16,4]$ :

$$
\frac{\mathscr{R}_{r s}}{\mathscr{R}_{x s} \mathscr{R}_{x r}}=c_{r} \sqrt{\left|\operatorname{det}\left(\boldsymbol{H}_{x s}+\boldsymbol{H}_{x r}\right)\right|},
$$

where $c_{r}$ is the velocity at receiver. 


\subsection{Kinematic two-point ray tracing}

To find all rays arriving at the receiver from a given source we use kinematic ray tracing [2]. Again we use the polar coordinate system in the source-receiver plane (Fig. 2):

$$
\begin{aligned}
& \frac{\mathrm{d} i}{\mathrm{~d} l}=\frac{\sin i}{c}\left(\frac{\mathrm{d} c}{\mathrm{~d} r}-\frac{c}{r}\right), \quad \frac{\mathrm{d} \phi}{\mathrm{d} l}=\frac{\sin i}{r}, \\
& \frac{\mathrm{d} r}{\mathrm{~d} l}=\cos i, \quad \frac{\mathrm{d} T}{\mathrm{~d} l}=\frac{1}{c}
\end{aligned}
$$

or

$$
\begin{aligned}
& \frac{\mathrm{d} i}{\mathrm{~d} r}=\tan i\left(\frac{1}{c} \frac{\mathrm{d} c}{\mathrm{~d} r}-\frac{1}{r}\right), \quad \frac{\mathrm{d} \phi}{\mathrm{d} r}=\frac{\tan i}{r}, \\
& \frac{\mathrm{d} l}{\mathrm{~d} r}=\frac{1}{\cos i}, \quad \frac{\mathrm{d} T}{\mathrm{~d} r}=\frac{1}{c \cos i} .
\end{aligned}
$$

Away from a discontinuity, the integration is done with ray distance $l$ as independent variable, using (19). Near a discontinuity, we use $r$ and (20) instead, such that the endpoint of the integration step coincides exactly with the discontinuity. At a discontinuity, continuity conditions are used:

$$
[r]_{-}^{+}=0, \quad[\sin i / c]_{-}^{+}=0
$$

where + and - represent the evaluation on the outgoing and incoming rays at the discontinuity depth, respectively. The second continuity condition is the Snel's law. A fourth-order Runge-Kutta method is used to solve (19) and (20).

The integration is started with a ray angle $i_{s}$ at the source. To find the $i_{s}$ from a source at a specified depth that has the ray hit the receiver, we pre-compute a table of $(i, \Delta)$ with the step size in $\Delta$ smaller than a specified precision (we found that $0.3^{\circ}$ works well with common global models such as AK135 or IASP91). We first find the interval $\Delta_{1}<\Delta^{*}<\Delta_{2}$ that brackets the receiver distance $\Delta^{*}$. An iterative linear interpolation scheme is then used to find $i_{s}$ :

$$
i_{k+1}=i_{k-1}+\frac{\Delta^{*}-\Delta_{k-1}}{\Delta_{k}-\Delta_{k-1}}\left(i_{k}-i_{k-1}\right) .
$$

This is repeated for every bracketing interval if there is more than one ray arriving at distance $\Delta^{*}$.

\subsection{Traveltime corrections}

We may apply corrections to the computed traveltimes to account for known deviation from spherical symmetry in the Earth, and bring the predicted time closer to that of a 3D Earth. The program computes the ellipticity, crustal, and topographic corrections of traveltimes, denoted by $\delta T_{\text {ell }}, \delta T_{\text {cru }}$ and $\delta T_{\text {top }}$, respectively. The traveltime after correction is

$$
T_{\text {cor }}=T_{\mathrm{BG}}+\delta T_{\text {ell }}+\delta T_{\text {cru }}+\delta T_{\text {top }}
$$

where $T_{\mathrm{BG}}$ is the predicted traveltime for the spherically symmetric background model.

\subsubsection{Crustal corrections}

The resolution length of global tomography is much larger than the wavelength of the 3D structures in the crust. Therefore, we apply crustal corrections based on a known crustal model instead of resolving the crustal structure in the tomography (inversion) part.

We denote traveltimes in the crust by lower case ' $t$ '. Crustal corrections are computed by first subtracting the traveltime in the background crust $\left(t_{\mathrm{BG}}\right)$, and then adding the traveltime in the known $3 \mathrm{D}$ crustal model $\left(t_{3 \mathrm{D}}\right)[12]:$

$$
\delta T_{\mathrm{cru}}=-t_{\mathrm{BG}}+t_{3 \mathrm{D}}
$$


For receivers and surface reflection points:

$$
t_{\mathrm{BG}}=\sum_{\text {segments }} \int_{r_{d}}^{a} \frac{|\cos i| \mathrm{d} r}{c_{\mathrm{BG}}}, \quad t_{3 \mathrm{D}}=\sum_{\text {segments }} \int_{r_{d}}^{a+h_{3 \mathrm{D}}} \frac{|\cos i| \mathrm{d} r}{c_{3 \mathrm{D}}} .
$$

For shallow sources in the crust with radius $r_{s}>r_{d}$ :

$$
t_{\mathrm{BG}}=\sum_{\text {segments }} \int_{r_{d}}^{r_{s}} \frac{|\cos i| \mathrm{d} r}{c_{\mathrm{BG}}}, \quad t_{3 \mathrm{D}}=\sum_{\text {segments }} \int_{r_{d}}^{r_{s}} \frac{|\cos i| \mathrm{d} r}{c_{3 \mathrm{D}}} .
$$

The significance of $r_{d}, a$, and $h_{3 \mathrm{D}}$ is explained in Fig. 3, which also illustrates the integral intervals. The sum is over all ray segments that are above $r_{d} . c_{\mathrm{BG}}$ and $c_{3 \mathrm{D}}$ are the velocities of the background model and the 3D crustal model, respectively. We use the 3D crustal model CRUST2.0 by Laske (available from http://mahi.ucsd.edu/Gabi/rem.dir/crust/crust2.html).

\subsubsection{Topographic corrections}

Even though model CRUST2.0 contains topography, the averaging over $2^{\circ} \times 2^{\circ}$ areas still leaves a residual topography for stations. The residual topography is small, so that the topographic correction can be approximated by

$$
\delta T_{\text {top }}=\left(h-h_{3 \mathrm{D}}\right) \frac{|\cos i|}{c_{3 \mathrm{D}}},
$$

where the angle $i$ and the velocity $c_{3 \mathrm{D}}$ are assumed constant and equal to their values at the surface. The significance of $h_{3 \mathrm{D}}$ and $h$ is explained in Fig. 3. Since the Fresnel zone for surface reflections (such as $P P$ or $S S$ ) is rather large, we assume that the average topography of CRUST2.0 is adequate to predict topography effects and we consider $h=h_{3 \mathrm{D}}$ for such reflections.

\subsubsection{Ellipticity corrections}

Following Dziewonski and Gilbert [5] we use Fermat's Principle to compute the effect of the elliptical perturbations on a ray in the spherical background model. The ellipticity correction is then the sum of contributions due to lengthening or shortening of the ray at its ends, displacements of discontinuities along the ray, and an integral over velocity perturbations $(\partial c / \partial r) \delta r$ due to the elliptical perturbation $\delta r$ :

$$
\delta T_{\text {ell }}=\frac{1}{p} \int_{0}^{\Delta} \frac{r^{3}}{c^{3}} \frac{\delta r}{r} \frac{\mathrm{d} c}{\mathrm{~d} r} \mathrm{~d} \phi \pm \sum_{d} \delta r\left(\frac{\cos i_{1}}{c_{1}}-\frac{\cos i_{2}}{c_{2}}\right),
$$

where $\phi$ is the polar coordinate in Fig. 2. The summation is over all discontinuities $d$, the + sign is to be used for transmitted rays and topside reflections like $P c P$, and the - sign is for bottomside reflections like $P P$. Index 1 is for the incoming, 2 for the outgoing ray.

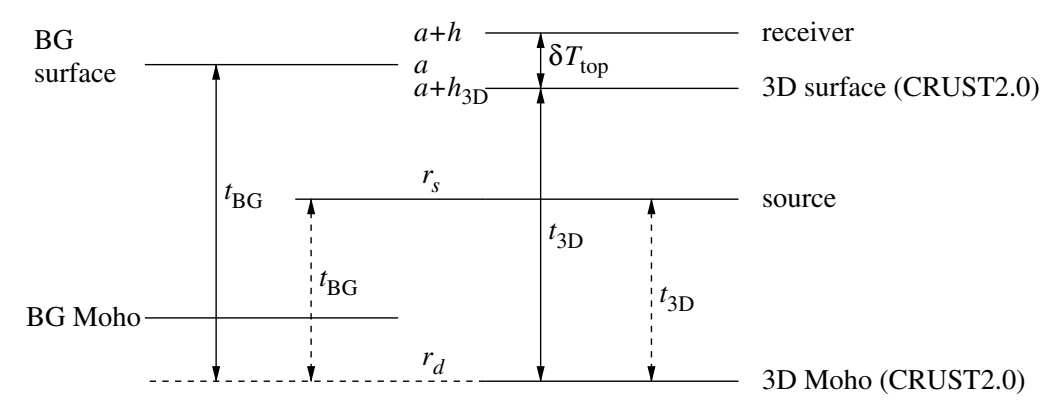

Fig. 3. Integral intervals (extended by arrows) for crustal and topographic correction calculation ((23)-(25)). 'BG' represents background model; '3D' represents the 3D crustal model. Solid arrows are for receivers and surface reflections; dashed arrows are for sources. The radius of each boundary is denoted, with $r_{d}$ being the deeper one of the background model Moho and the 3D crust Moho, $r_{s}$ the source radius, $a$ the sea level of the background model $(6371 \mathrm{~km}), h_{3 \mathrm{D}}$ the elevation of the $3 \mathrm{D}$ crust surface, and $h$ the elevation of the receiver. 


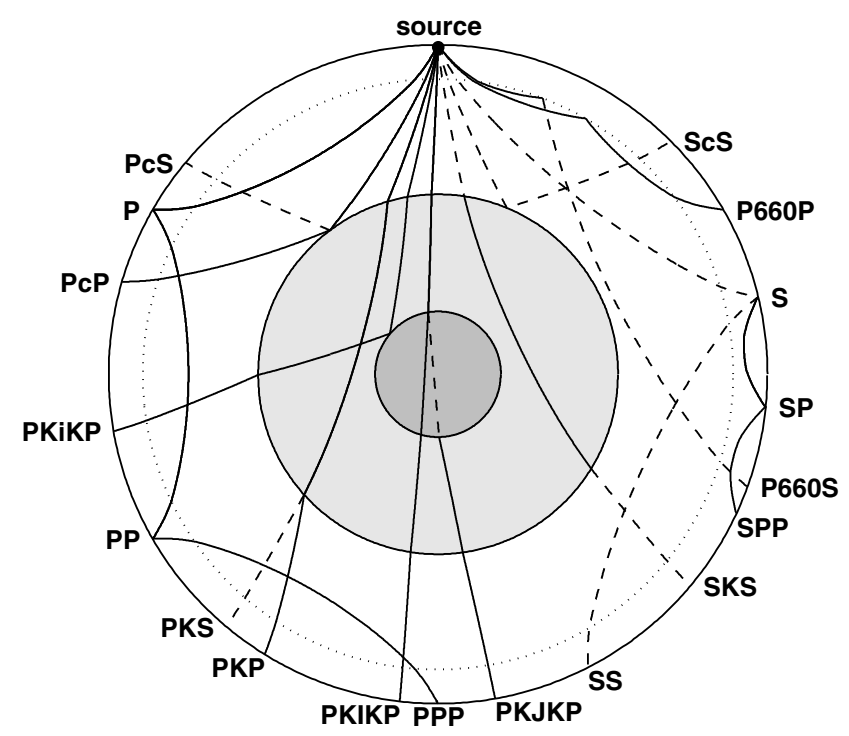

Fig. 4. Ray paths of global phases produced by the kinematic ray tracing of the program, for a 5-km-deep earthquake. The shaded areas (from dark to white) represent inner core, outer core, and mantle. The dotted circle indicates the $660 \mathrm{~km}$ discontinuity. Solid lines represent $P$ waves, and dashed lines represent $S$ waves.

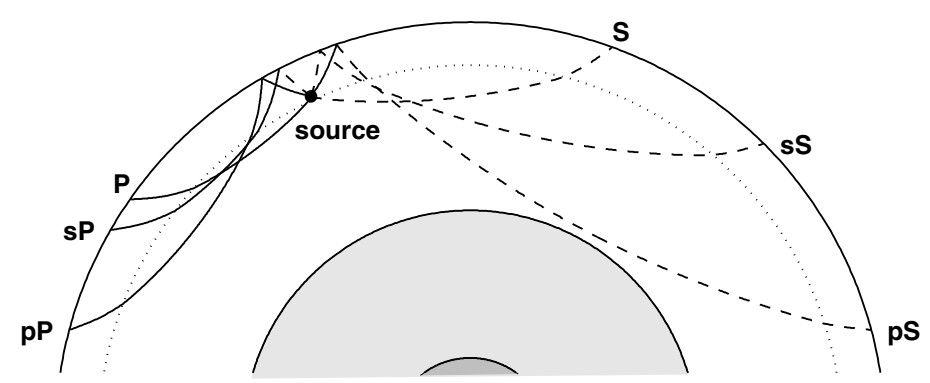

Fig. 5. Ray paths of depth phases produced by the kinematic ray tracing of the program, for a $600-\mathrm{km}$-deep earthquake. Notations are the same as in Fig. 4.

The deflection $\delta r$ is a function of depth and latitude:

$$
\frac{\delta r}{r} \approx \epsilon(r)\left(\frac{1}{3}-\cos ^{2} \theta\right)
$$

where $\epsilon(r)$ is the ellipticity of the Earth. When tracing the ray, the value of $\cos \theta \operatorname{can}$ be found if we realize that it is simply the $z$-component of the unit vector $\hat{\boldsymbol{r}}$ to that point:

$$
\hat{\boldsymbol{r}}=\hat{\boldsymbol{r}}_{p} \sin \phi+\hat{\boldsymbol{r}}_{s} \cos \phi .
$$

$\hat{\boldsymbol{r}}_{p}$ is the vector in the ray plane orthogonal to the source unit vector $\hat{\boldsymbol{r}}_{s}$ :

$$
\hat{\boldsymbol{r}}_{p}=\frac{\hat{\boldsymbol{r}}_{r}-\hat{\boldsymbol{r}}_{s} \cos \Delta}{\left|\hat{\boldsymbol{r}}_{r}-\hat{\boldsymbol{r}}_{s} \cos \Delta\right|}
$$

and $\hat{\boldsymbol{r}}_{r}$ is the receiver unit vector, $\Delta=\cos ^{-1}\left(\hat{\boldsymbol{r}}_{s} \cdot \hat{\boldsymbol{r}}_{r}\right)$. This approach differs from that given by Dziewonski and Gilbert, who express $\delta r$ in terms of associated Legendre functions. Our algorithm is more efficient when one is actually tracing rays. 
(a) $\mathbf{P}$

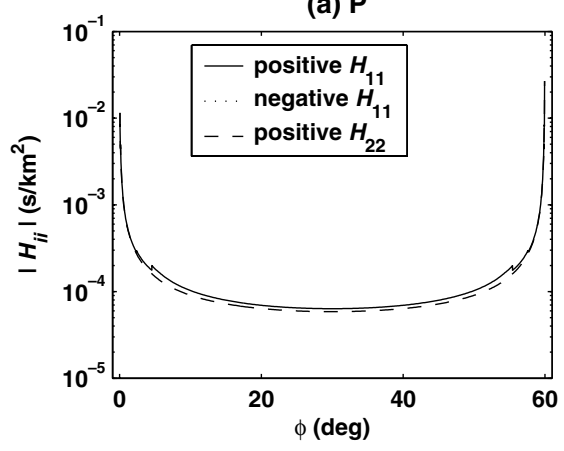

(c) PcP

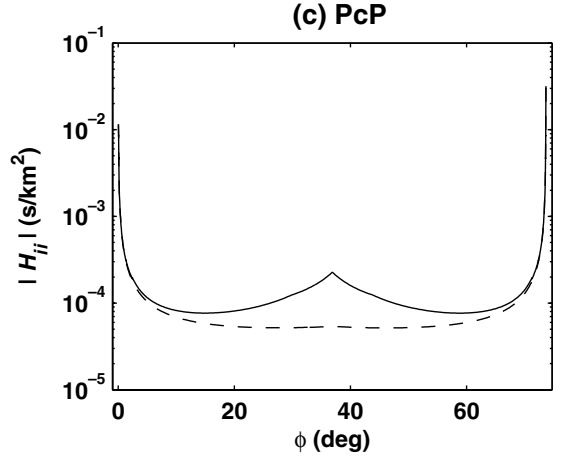

(b) PP

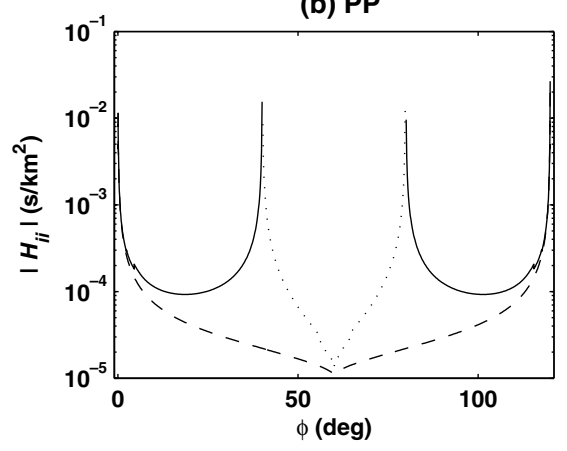

(d) PKP

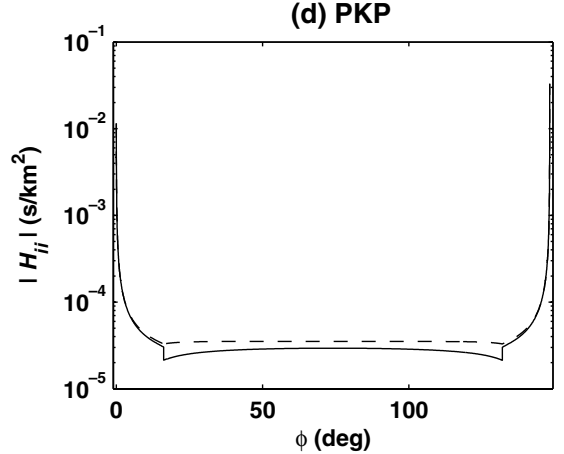

Fig. 6. Elements of Hessian matrix $\boldsymbol{H}=\boldsymbol{H}_{x s}+\boldsymbol{H}_{x r}=\operatorname{diag}\left(H_{11}, H_{22}\right)$ for different $P$ phases. $\phi$ is the polar coordinate (Fig. 2). Divergence occurs at source, receiver, and caustics (see (b)). Caustics also change the sign of $H_{11}$. The small jumps of $H_{11}$ in $P$ and $P P$ are due to the $660 \mathrm{~km}$ discontinuity. The small jumps of $H_{11}$ in $P K P$ are due to the core-mantle boundary.

\subsubsection{Dispersion corrections}

A direct byproduct of kinematic ray tracing is that it enables us to compute $t^{*}$ :

$$
t^{*}=\int_{0}^{L} \frac{\mathrm{d} l}{c Q}
$$

where the integration is along the ray path and $Q$ is the quality factor. $t^{*}$ can be used to correct traveltimes for dispersion by using

$$
\delta T_{\mathrm{dis}}=-\frac{t^{*}}{\pi} \ln \left(\frac{\omega}{\omega_{0}}\right)
$$

where $\omega_{0}$ is the reference frequency for the velocity model. If data are collected in different frequency bands, this dispersion correction must be added to the corrections in (21).

\section{Numerical tests}

To examine the validity and generality of the program, we performed extensive numerical tests for different source and receiver locations, different phases, and different background models. This section discusses the major results of these tests. Since computational errors for $P$ waves are generally smaller than those for $S$ waves, our emphasis will be 'worst case', i.e. mostly on $S$ waves.

\subsection{Ray tracing examples}

The most important function of the program is the kinematic and dynamic ray tracing in the 1D background model. Our goal is to make the ray tracing accurate for all imaginable phases. The program accepts 
(a) $\mathrm{S}$

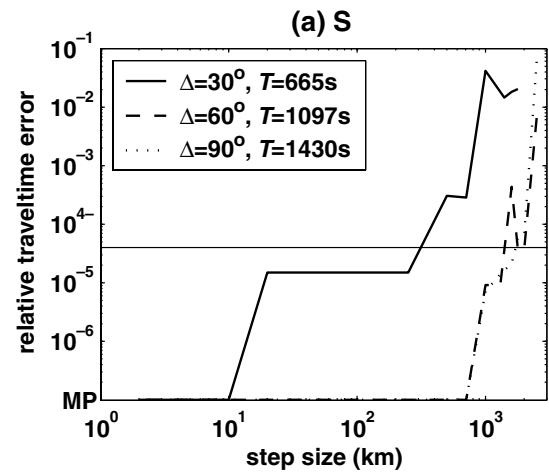

(c) ScS

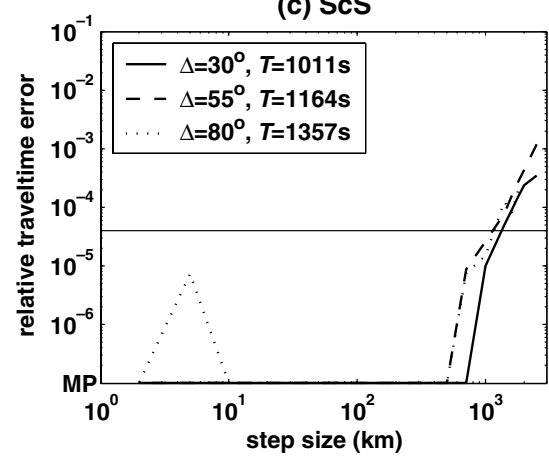

(b) SS

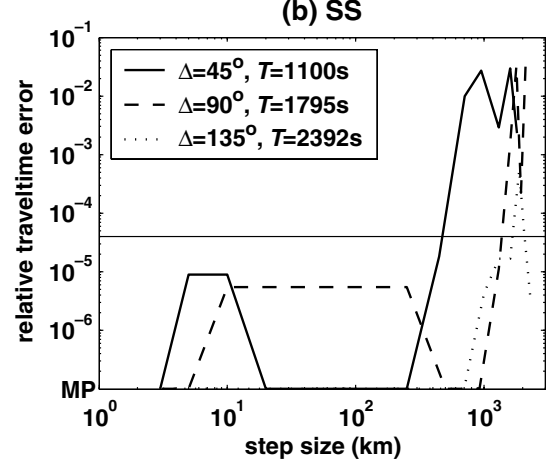

(d) SKS

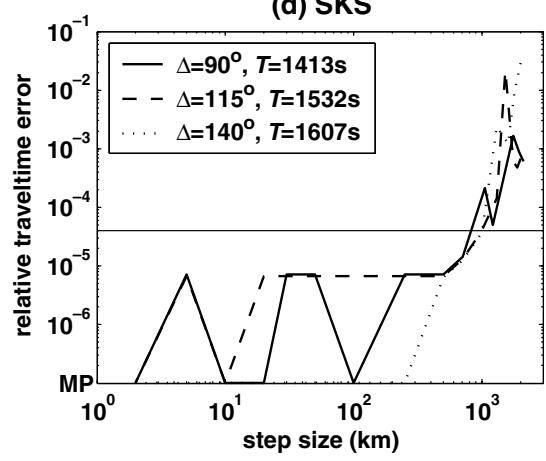

Fig. 7. Convergence of the traveltime with decreasing step size in kinematic ray tracing. The vertical axis is defined by (27). 'MP' represents machine precision, i.e. the smallest positive number on the computer $(2 \mathrm{e}-16)$. The horizontal solid line indicates the error tolerance level determined by a traveltime error of $0.1 \mathrm{~s}$. The model used is IASP91. Three examples are shown for each phase, with epicentral distances and traveltimes (of smallest step size) indicated.

as input a user-defined ray trajectory. Ray types are defined by segments that specify the starting depth of the segment, the nature of the starting point (source, reflection, transmission or turning point) the type of wave ( $P$ or $S$ ), and the number of polar passages, which may be $>0$ for waves such as $P P P$ for which $\Delta>180^{\circ}$. This codification of rays gives maximum flexibility in the software to accept all possible rays. Figs. 4 and 5 show the ray paths of different phases produced by the kinematic ray tracing of the program, for a shallow earthquake and a deep earthquake, respectively. Such figures served as a first global check on the correctness of the ray tracing code.

Fig. 6 shows the diagonal elements of the Hessian matrix

$$
\boldsymbol{H}=\boldsymbol{H}_{x s}+\boldsymbol{H}_{x r}=\operatorname{diag}\left(H_{11}, H_{22}\right)
$$

for different $P$ phases. $S$ phases have similar curves but smaller jumps. By definition (5), $H_{i i}=\partial^{2} T / \partial q_{i}^{2}$, so its physical meaning is the curvature in the $q_{i}$ direction of the wavefront defined by a constant travel time $T(\boldsymbol{r})$. At the source, since the radius of the forward wavefront is zero, $\boldsymbol{H}_{x s}$ goes to infinity. Similarly, $\boldsymbol{H}_{x r}$ goes to infinity at receiver, as we have seen in Section 3.2. The divergent properties of $\boldsymbol{H}$ at source and receiver are seen for all phases in Fig. 6. Another divergent point with infinite wavefront curvature is found at the caustic, where a ray bundle in the source-receiver plane converges to a single line. $P P$ waves have caustics, which appear as the two middle spikes in Fig. 6b. Caustics also change the sign of $H_{11}$, because when passing through a caustic, the wavefront changes from convex to concave (or from concave to convex) in the $\boldsymbol{q}_{1}$ direction. $H_{22}$ is not influenced by caustics, except at the antipode, which is a rare occasion. The small jumps of $H_{11}$ in $P K P$ visible in Fig. 6d are due to the core-mantle boundary discontinuity. The small jumps of $H_{11}$ in $P$ and $P P$ are due to the $660 \mathrm{~km}$ discontinuity; these jumps exist for $P c P$ and $P K P$ but are invisible at the scale of the figure. $H_{22}$ does not show jumps because there is no discontinuity in the $\boldsymbol{q}_{2}$ direction (perpendicular to the source-receiver plane) for a 1D Earth. 
(a) $\mathrm{S}$

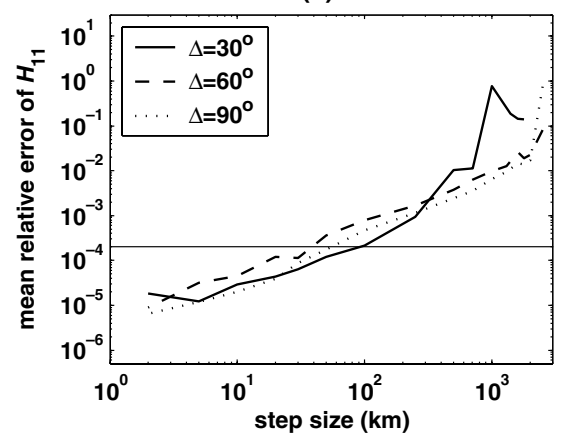

(c) ScS

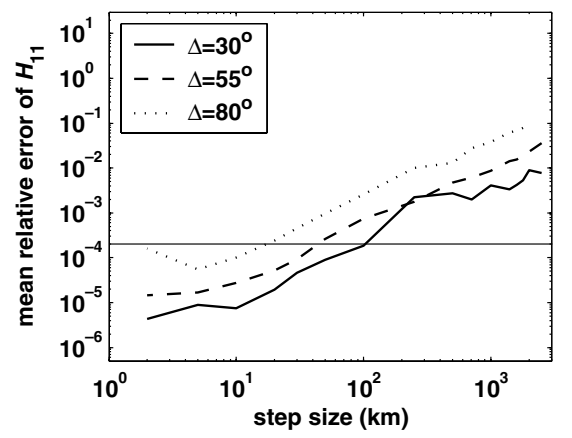

(b) SS

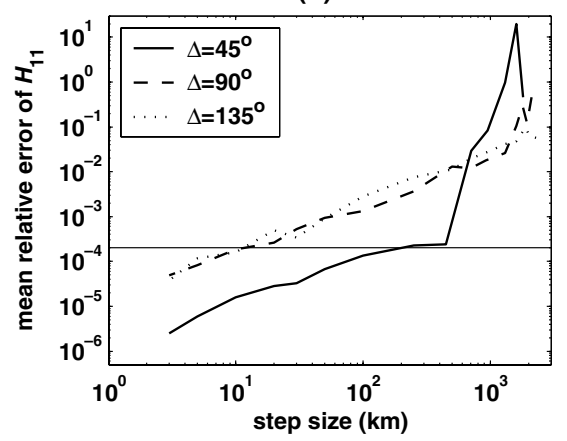

(d) SKS

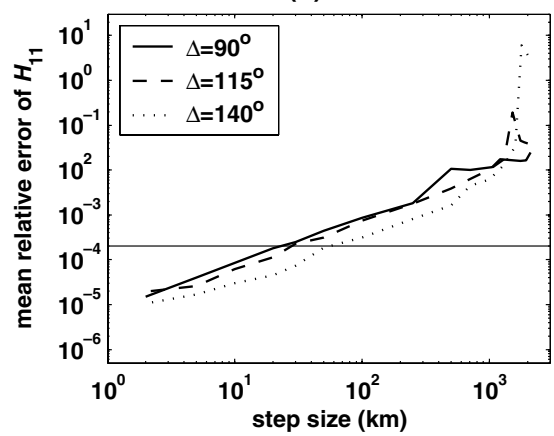

Fig. 8. Convergence of $H_{11}$ with decreasing step size in kinematic ray tracing. The vertical axis is the mean of $e_{H_{11}}$ (see (31)) over the ray. Three examples with different epicentral distances are shown for each phase. The horizontal solid line indicates the error tolerance level determined by a detour time error of $0.1 \mathrm{~s}$.

\subsection{Validation of traveltime computation}

Validation of traveltimes is surprisingly difficult. Direct comparison with existing codes is of little use because of differences in interpolation between the model nodes, which leads to small differences in travel time. Allowing for such differences, the traveltimes computed agree well with those tabled for models IASP91 [9] and AK135 [10], but this is insufficient as a test.

A good way to validate the traveltime computation in the kinematic ray tracing, which at the same time gives us insight in the influence of the step size $b$ used in the integration of (19), is to examine its convergent behavior with decreasing $b$. We define a relative traveltime error estimate

$$
e_{T}=\frac{|\mathrm{d} T|}{T}=\frac{\left|T(b)-T\left(b_{\min }\right)\right|}{T\left(b_{\min }\right)},
$$

where $b_{\min }$ is the smallest step size. Some results are shown in Fig. 7, where the horizontal line represents a tolerable error level $\left(0.1 \mathrm{~s}\right.$ at $\Delta=30^{\circ}$, which is well below the typical observational uncertainty). The model used is IASP91. It is clear that $e_{T}$ converges to a level well below the error tolerance for $b<300 \mathrm{~km}$, which is strong evidence that the traveltime computation is accurate. We note that the fourth-order Runge-Kutta method samples the model at steps $b / 2$, and that the actual step size in the crust and upper mantle is limited to the distance between discontinuities. We can also see that $T$ converges more slowly for a smaller epicentral distance (Fig. 7a and b), partly because the ratio of $b$ to the total ray length is larger, but mostly because a shallow ray is much more strongly influenced by more rapidly varying upper mantle structure.

\subsection{Validation of Hessian matrix computation}

Several analytical results for elements of the Hessian matrix (26) are available ([4]): 
(a) $\mathrm{S}$

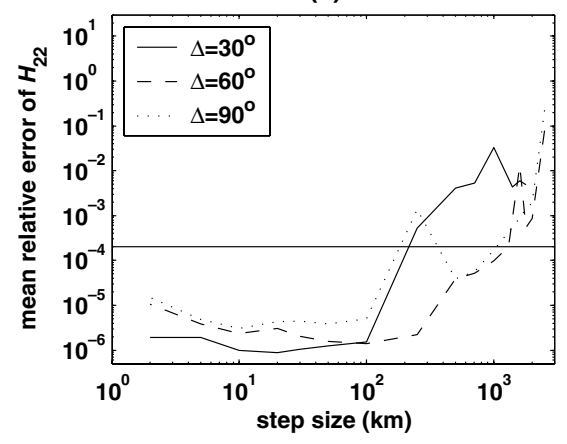

(c) ScS

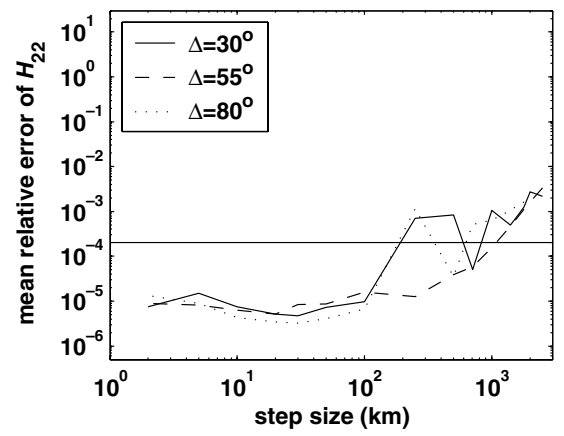

(b) SS

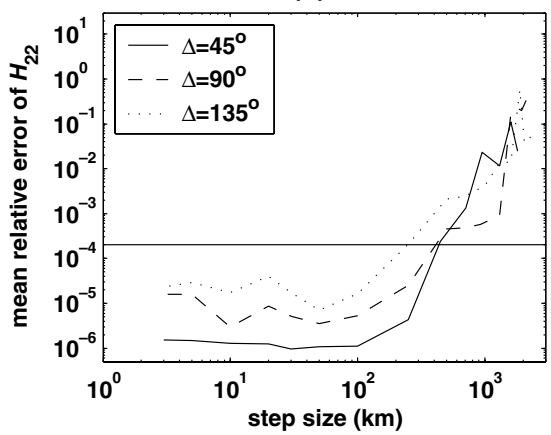

(d) SKS

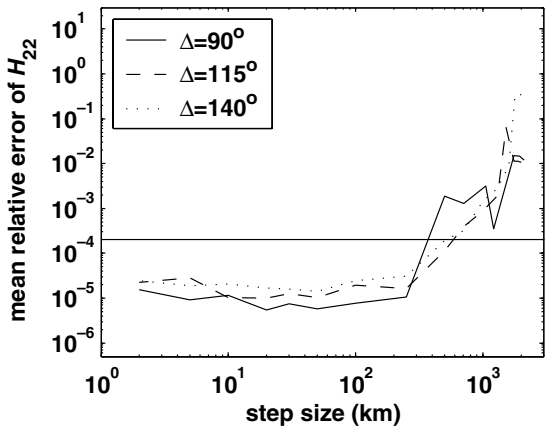

Fig. 9. Convergence of $H_{22}$ with decreasing step size in kinematic ray tracing. The vertical axis is the mean of $e_{H_{22}}$ (see (32)) over the ray. Three examples with different epicentral distances are shown for each phase. The horizontal solid line indicates the error tolerance level determined by a detour time error of $0.1 \mathrm{~s}$.

$$
\begin{aligned}
& f_{1} \equiv r \cos i P_{1}+\left(\frac{p^{2}}{r} \frac{\mathrm{d} c}{\mathrm{~d} r}-\frac{1}{c}\right) Q_{1}-r_{s} \cos i_{s}=0, \\
& f_{2} \equiv r \cos (\pi-i) P_{3}+\left(\frac{p^{2}}{r} \frac{\mathrm{d} c}{\mathrm{~d} r}-\frac{1}{c}\right) Q_{3}-r_{r} \cos \left(\pi-i_{r}\right)=0, \\
& f_{3} \equiv \frac{p \sin \Delta}{r^{2} \sin \phi \sin (\Delta-\phi)}=H_{22} .
\end{aligned}
$$

Since $H_{11}=P_{1} / Q_{1}+P_{3} / Q_{3}$ (see (11), (16)), (28) and (29) can be used to check the numerical precision of $H_{11}$. Let

$$
e_{H_{11}}=\left|\frac{f_{1}}{r_{s} \cos i_{s}}\right|+\left|\frac{f_{2}}{r_{r} \cos \left(\pi-i_{r}\right)}\right| \text {. }
$$

$e_{H_{11}}$ is of the same order as $\mathrm{d} H_{11} / H_{11}$ and thus a good estimate of the numerical error of $H_{11}$. Eq. (30) could be used to eliminate the Runge-Kutta integration for $H_{22}$ altogether, but has so far been kept because it provides an independent check on correctness of the code and the influence of round-off errors. The numerical error of $H_{22}$ is

$$
e_{H_{22}}=\left|\frac{H_{22}-f_{3}}{f_{3}}\right| .
$$

Figs. 8 and 9 plot the convergence of the average $e_{H_{11}}$ and $e_{H_{22}}$ (over the ray) with decreasing step size used to integrate (19), using the same examples as in Fig. 7. The horizontal solid line indicates a rough estimate of the error tolerance level, which gives a detour time error estimate of $0.1 \mathrm{~s}$. Deviation from the quadratic dependence (3) is of course not included in this error. It can be seen that $e_{H_{22}}$ converges fast and well below the error 

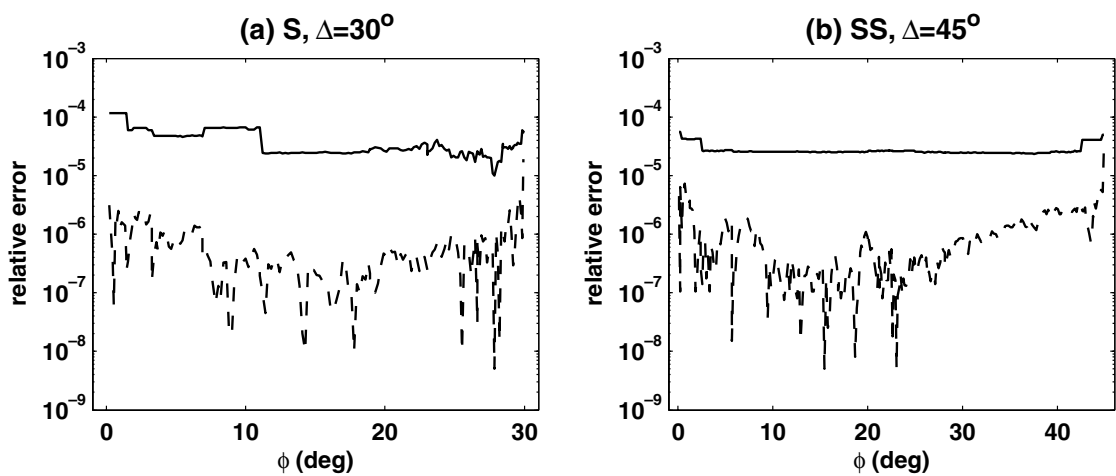

(c) ScS, $\Delta=30^{\circ}$
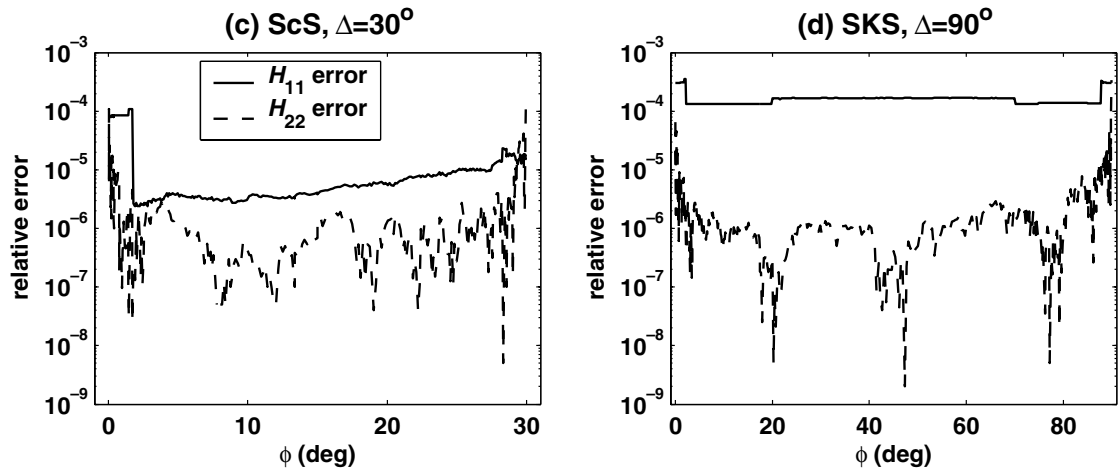

Fig. 10. Relative numerical errors of $H_{11}, H_{22}$ along the ray paths for different phases. The solid line represents $e_{H_{11}}$ in (31), the dashed line represents $e_{H_{22}}$ in (32), and $\phi$ is the polar coordinate in Fig. 2. The step size in kinematic ray tracing is $20 \mathrm{~km}$ for each phase.

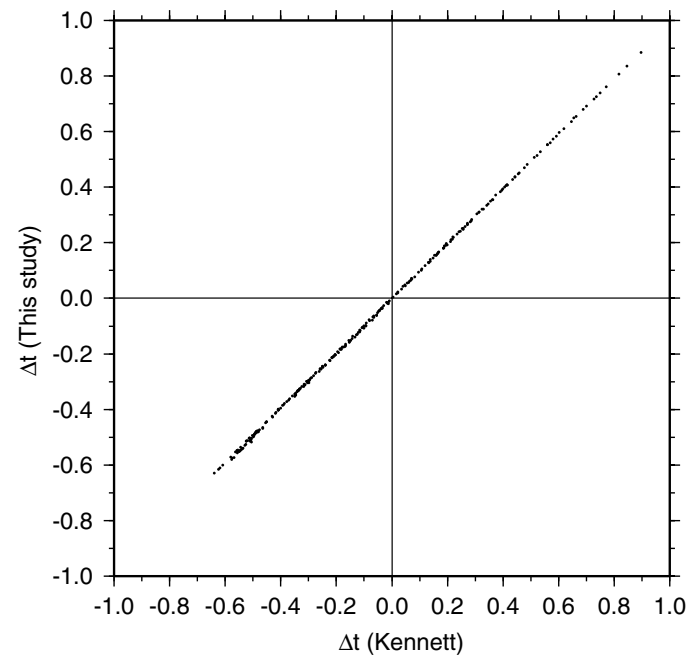

Fig. 11. Ellipticity corrections (in sec, for $P$ waves) computed by this study (vertical axis) and the program ellip.f by Kennett [11] (horizontal axis).

tolerance level (Fig. 9), while $e_{H_{11}}$ converges relatively slowly and to a relatively high error (but still below the error tolerance level, Fig. 8). The optimum step size, which is as large as possible while keeping the error below the tolerance level, is thus determined by the behavior of $e_{H_{11}}$. From Fig. 8, the optimum step size is about $30 \mathrm{~km}$ for $S$ waves, $20 \mathrm{~km}$ for $S c S$ and $S K S$ waves, and $10 \mathrm{~km}$ for $S S$ waves. The fact that $e_{H_{11}}$ is larger than $e_{H_{22}}$ may be explained by the following. The numerical errors of $H_{11}$ and $H_{22}$ are due to the numerical integration of (12) and the application of continuity conditions (14). At every discontinuity, the application of (14) 

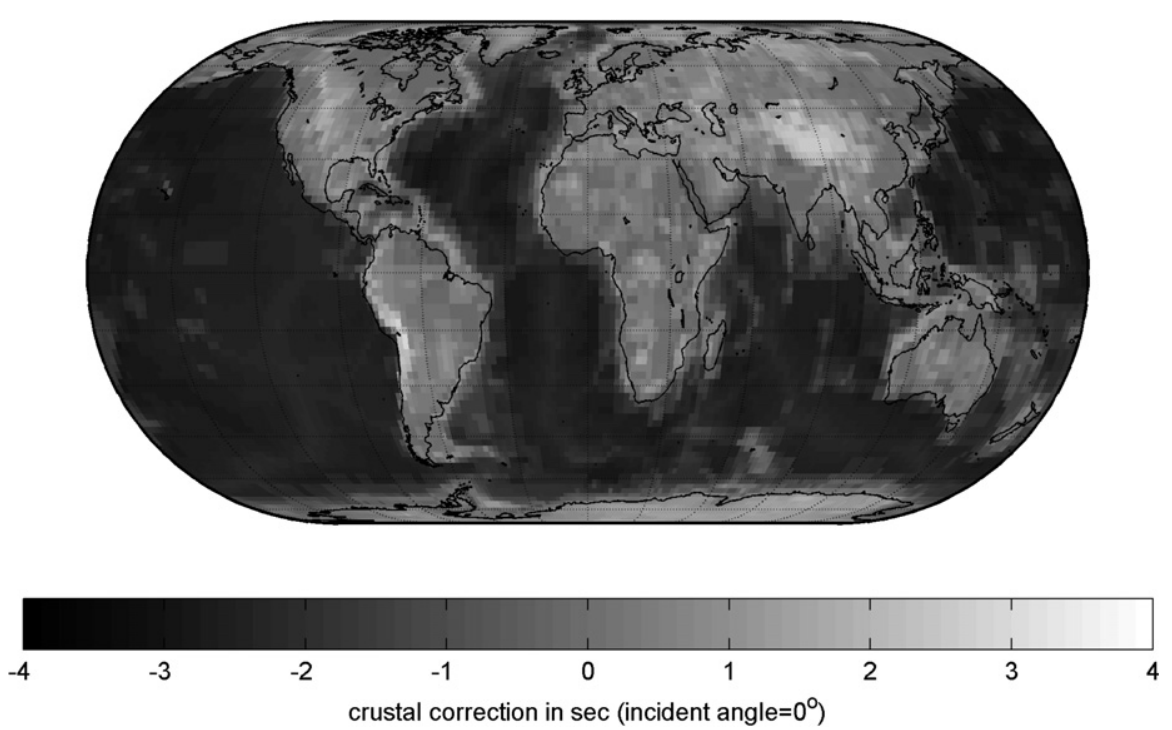

Fig. 12. Map of crustal corrections $\delta T_{\text {cru }}$ through the entire crust for vertical $S$ waves.
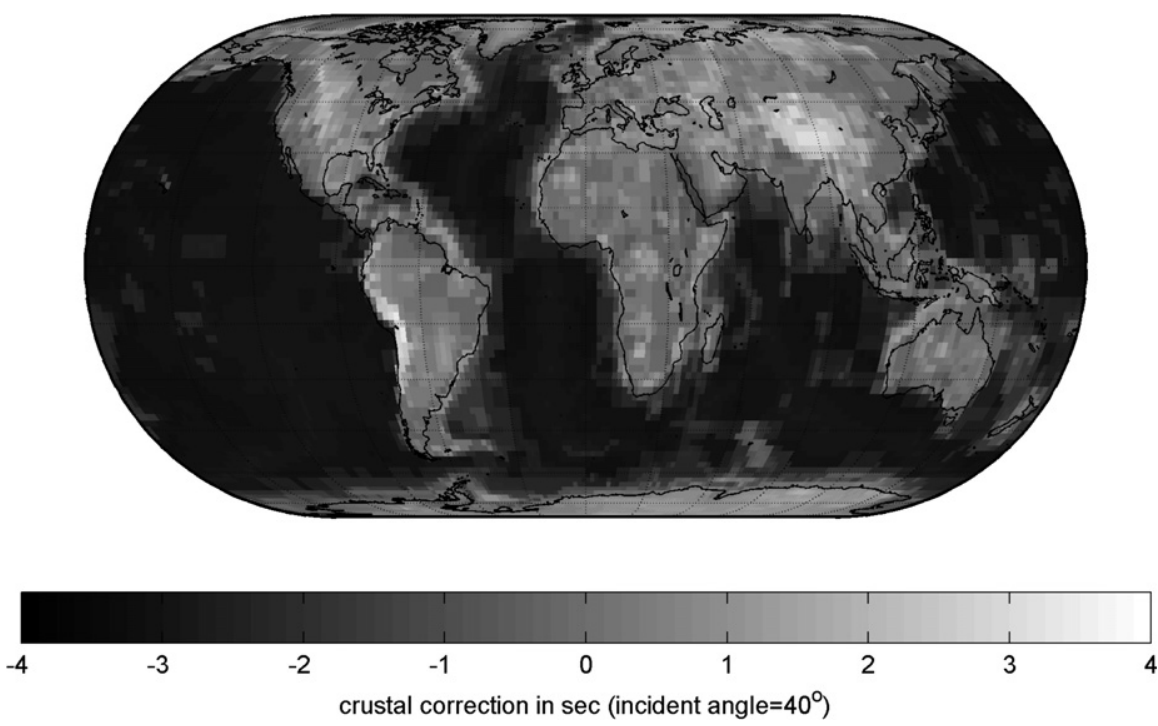

Fig. 13. Map of crustal corrections $\delta T_{\text {cru }}$ through the entire crust for $S$ waves with an incident angle of $40^{\circ}$ at background model surface.

gives numerical errors to all $P$ 's and $Q$ 's except $Q_{2}, Q_{4}$, so $H_{22}=P_{2} / Q_{2}+P_{4} / Q_{4}$ has smaller numerical errors than $H_{11}=P_{1} / Q_{1}+P_{3} / Q_{3}$. It can also be seen from (12) and (14) that the $P_{1}, Q_{1}, P_{3}, Q_{3}$-related formula are more complicated than the $P_{2}, Q_{2}, P_{4}, Q_{4}$-related formula, which enhances the chance of a larger numerical error of $H_{11}$.

Fig. 10 provides a detailed examination of $e_{H_{11}}$ and $e_{H_{22}}$ along a particular ray path. Again, we see that $e_{H_{11}}$ is 1-2 orders of magnitude larger than $e_{H_{22}}$, as discussed before. The numerical errors increase toward source and receiver, due to the divergent properties of $\boldsymbol{H}$ at source and receiver.

\subsection{Validation of traveltime corrections}

The ellipticity corrections are tested by comparison to the output of program ellip.f by Kennett [11], which interpolates tables of $\delta T_{\text {ell }}$. For $P, P P$, and $P c P$ waves, absolute differences are at most $0.01,0.02$ 
and $0.04 \mathrm{~s}$, respectively (Fig. 11), which is acceptable in view of different models for the ellipticity (we used the values from Huang et al. [6]).

Figs. 12 and 13 are maps of crustal corrections $\delta T_{\text {cru }}$ in (22) through the entire crust for $S$ waves with zero and steep incident angles, respectively. Fig. 12 is representative of global phases and Fig. 13 is representative of regional phases (a surface incident angle of $40^{\circ}$ corresponds to an epicentral distance less than $15^{\circ}$ ). For both cases, continents have positive $\delta T_{\text {cru }}$ and oceans have negative $\delta T_{\text {cru }}$. This is understandable because continental crust is thick and relatively slow (especially sedimentary layers), and thus $t_{3 \mathrm{D}}>t_{\mathrm{BG}}$ in (22). On contrary, oceanic crust is thin and relatively fast (e.g. basalt), so $t_{3 \mathrm{D}}<t_{\mathrm{BG}}$. At a first glance, it is surprising that the magnitude of $\delta T_{\text {cru }}$ in Fig. 13 is larger than that in Fig. 12, considering the factor of $\cos i$ in (23). However, for the same ray parameter, the incident angle in background model $i_{\mathrm{BG}}$ is different from the incident angle in 3D crust $i_{3 \mathrm{D}}$, due to the different velocities. Taking Tibet as an example, a surface $i_{\mathrm{BG}}=40^{\circ}$ corresponds to a surface $i_{3 \mathrm{D}}=13^{\circ}$, and thus a larger $t_{3 \mathrm{D}}$.

\section{Conclusions}

A program raydyntrace.f for dynamic ray tracing in arbitrary models of the Earth for arbitrary ray configurations has been tested on a number of frequently observed seismic phases. An accuracy of $0.1 \mathrm{~s}$ in traveltimes and detour times is easily obtained, which is well within the demands imposed by typical observational uncertainties. In addition to providing the parameters needed to compute geometrical spreading and paraxial ray traveltimes, the program computes traveltime corrections for crustal structure, topography, and ellipticity, and computes attenuation such as $t^{*}$.

\section{Acknowledgments}

We thank Ignace Loris for discussion. Yue Tian was supported by the National Science Foundation under grant number EAR0309298.

\section{References}

[1] M. Calvet, S. Chevrot, Traveltime sensitivity kernels for PKP phases in the mantle, Phys. Earth Planet. Int. 153 (2005) $21-31$.

[2] F. Dahlen, J. Tromp, Theoretical Global Seismology, Princeton University Press, 1998.

[3] F.A. Dahlen, A.M. Baig, Fréchet kernels for body-wave amplitudes, Geophys. J. Int. 150 (2002) 440-466.

[4] F.A. Dahlen, S.-H. Hung, G. Nolet, Fréchet kernels for finite-frequency traveltimes - I. Theory, Geophys. J. Int. 141 (2000) 157174.

[5] A.M. Dziewonski, F. Gilbert, The effect of small, aspherical perturbations on travel times and a re-examination of the corrections for ellipticity, Geophys. J. Roy. Astr. Soc. 44 (1976) 7-17.

[6] C. Huang, W. Jin, X. Liao, A new nutation model of a non-rigid earth with ocean and atmosphere, Geophys. J. Int. 146 (2001) 126133.

[7] S.-H. Hung, F.A. Dahlen, G. Nolet, Fréchet kernels for finite-frequency traveltimes - II. examples, Geophys. J. Int. 141 (2000) 175203.

[8] S.-H. Hung, F.A. Dahlen, G. Nolet, Wavefront healing: a banana-doughnut perspective, Geophys. J. Int. 146 (2001) $289-312$.

[9] B. Kennett, E. Engdahl, Travel times for global earthquake location and phase association, Geophys. J. Int. 105 (1991) $429-465$.

[10] B. Kennett, E. Engdahl, R. Buland, Constraints on seismic velocities in the earth from traveltimes, Geophys. J. Int. 122 (1995) 108124.

[11] B. Kennett, O. Gudmundsson, Ellipticity corrections for seismic phases, Geophys. J. Int. 127 (1996) 40-48.

[12] R. Montelli, G. Nolet, F.A. Dahlen, G. Masters, A catalogue of deep mantle plumes: new results from finite-frequency tomography, Geochem. Geophys. Geosyst 7 (Q11007) (2006), doi:10.1029/2006GC001248.

[13] R. Montelli, G. Nolet, F.A. Dahlen, G. Masters, E.R. Engdahl, S.-H. Hung, Finite-frequency tomography reveals a variety of plumes in the mantle, Science 303 (2004) 338-343.

[14] R. Montelli, G. Nolet, G. Masters, F.A. Dahlen, S.-H. Hung, Global $P$ and $P P$ traveltime tomography: rays versus waves, Geophys J. Int. 158 (2004) 637-654.

[15] G. Nolet, F.A. Dahlen, R. Montelli, Traveltimes and amplitudes of seismic waves: a re-assessment, in: A. Levander, G. Nolet (Eds.), Array Analysis of Broadband Seismograms, AGU Monograph Series, 2005, pp. 37-48.

[16] R. Snieder, C.H. Chapman, The reciprocity properties of geometrical spreading, Geophys. J. Int. 150 (1998) $440-466$.

[17] J. Tromp, C. Tape, Q. Liu, Seismic tomography, adjoint methods, time reversal and banana-doughnut kernels, Geophys. J. Int. 160 (2005) 195-216. 
[18] V. Červený, The application of ray tracing to the numerical modeling of seismic wavefields in complex structures, in: G. Dohr (Ed.), Seismic Shear Waves, Part A: Theory, Handbook of Geophysical Exploration, 15A, Geophysical Press, London, 1985 , pp. 1-124.

[19] V. Červený, F. Hron, The ray series method and dynamic ray tracing systems for 3-D inhomogeneous media, Bull. Seis. Soc. Am. 70 (1980) 47-77. 